\title{
Trocar-Site Herniation (TSH) of the Small Bowel following Laparoscopy Despite Rectus Sheath Closure, Resulting in Subacute Bowel Obstruction in the Early Post-Operative Period: A Case Discussion and Review of Literature
}

\author{
Senadheera, DI*, Jayasundara, DMCS and Jayawardane, IA \\ De Soysa Hospital for Women, Colombo, Sri Lanka \\ *Corresponding author: Senadheera, De Soysa Hospital for Women, Colombo, Sri Lanka
}

\section{Abstract}

Laparoscopy is becoming the gold slandered of access to the abdomen and pelvis. However, it's not without unique complications. Trocar site hernia is a rare but a serious complication which can cause diagnostic confusion, in the early post-operative period. It can be potentially life threatening with obstruction, strangulation, incarceration and finally perforation of the bowel, after a seemingly uncomplicated laparoscopy. First ever TSH, reported by "Fear in 1968". Early studies described incidence from $0.002 \%$ to $5.2 \%$. With improved surgical techniques \& new closure methods, incidence has gone down significantly. Recent study, analyzing 55,244 cases reported it to be $0.016 \%$. We discuss a case of small bowel herniation through the " $12 \mathrm{~mm}$ lateral port", even after aponeurotic closure with polyglactin. She presented with subacute bowel obstruction needing laparotomy. A diagnostic dilemma can arise in differentiating TSH, from "iatrogenic bowel perforation \&peritonitis". This article discusses how to minimize this serious complication, different port closure methods, suture materials, using currently available evidence and recommendations. USS may be less convincing particularly in obese. Diagnosis is commonly aided by plain X-ray film. CECT has significant advantages in differentiating TSH from incarcerated small bowel \& ileus. RCOG advices closure of all $7 \mathrm{~mm}$ or larger lateral ports \& $10 \mathrm{~mm}$ or larger midline ports. It can be prevented with proper pre-op evaluation, appropriate port selection, good specimen retrieval techniques, and use of proper closure methods \& devices. Conventional J needle, laparoscopic suturing of the rectus sheath under direct vision, port closure devices, all have their own pros \& cons. Specially designed port closure devices are encouraged wherever possible, especially in obese as well as patience
\end{abstract}

with other risk factors. European hernia society recommends slowly absorbable monofilament sutures. Suture materials with higher tensile strength such as PDS in comparison to polyglactin may further improve outcome.

\section{Introduction}

Since, the acclaimed gynecologist Dr. Harry Reich described the first laparoscopic hysterectomy in 1989, the novel surgical technique has contributed for remarkable improvements in gynaecological practice [1]. Laparoscopy is becoming the gold slandered mode of access to the abdominal cavity and pelvis, such as in endometriosis and ectopic pregnancy with high patient satisfaction, enhanced recovery times and minimal complications. However laparoscopic surgery is not without unique complications of its own. Trocar site hernia is a rare but a serious complication which can cause diagnostic confusion in the early post-operative period [2]. It can be potentially life threatening with obstruction, strangulation, incarceration and finally perforation of the bowel, especially with delayed diagnosis. First ever port site hernia was reported by "Fear in 1968" in his gynecological laparoscopy case series [3]. Early studies described incidence ranging from $0.002 \%$ to $5.2 \%$ [4]. With the improvement of surgical techniques, development of new closure methods, incidence has gone down significantly. Recent study, analyzing 55,244 cases reported it to be $0.016 \%$ [5]. Here we discuss a case diagnosed to

Citation: Senadheera, Jayasundara, Jayawardane (2020) Trocar-Site Herniation (TSH) of the Small Bowel following Laparoscopy Despite Rectus Sheath Closure, Resulting in Subacute Bowel Obstruction in the Early Post-Operative Period: A Case Discussion and Review of Literature. Obstet Gynecol Cases Rev 7:186. doi.org/10.23937/2377-9004/1410186

Accepted: December 10, 2020: Published: December 12, 2020

Copyright: (c) 2020 Senadheera, et al. This is an open-access article distributed under the terms of the Creative Commons Attribution License, which permits unrestricted use, distribution, and reproduction in any medium, provided the original author and source are credited. 
have small bowel loop herniation through the "12 mm lateral port entry site", even after sheath closure with polyglactin sutures. She presented with subacute bowel obstruction needing laparotomy in the early post-operative period following laparoscopic surgery. A diagnostic dilemma can arise in differentiating TSH, from "iatrogenic bowel perforation \& peritonitis" specially, with mixed clinical symptoms and rising inflammatory markers. We discuss the improvements that can be made to minimize this serious complication, \& also the different port closure methods, different suture materials, using currently available best evidence and recommendations.

\section{Case Presentation}

A 34-year-old lady (gravida 1 para 0) who had undergone a laparotomy for ruptured ectopic pregnancy, presented to us with a history of secondary subfertility for 4 years. Her body mass index was $26 \mathrm{~kg} / \mathrm{m}^{2}$. She was complaining of cyclical dysmenorrhea deep dyspareunia \& chronic pelvic pain suggestive of endometriosis. Pre-operative USS assessment revealed left sided Endo- metrioma with bi-lateral kissing ovaries. Type 4 subserosal fibroid measuring $3 \mathrm{~cm}$ by $4 \mathrm{~cm}$ was visible in close proximity to right uterine cornua. After a careful evaluation, she underwent laparoscopic cystectomy, adhesiolysis and myomectomy with tubal patency testing.

She had Palmers entry, followed by three $5 \mathrm{~mm}$ lateral ports and $12 \mathrm{~mm}$ supraumbilical central port (Port placements are shown in Figure 1). Palmers entry was chosen due to expected bowel adhesions with the history of severe endometriosis and previous laparotomy. All trocars used were the non-bladed, sharp pyramidal type. Intraoperative findings were suggestive of grade 4 endometriosis with a pedunculated fibroid close to the tube. She underwent extensive pelvic adhesiolysis \& resection of endometriosis, cystectomy of the endometrioma \& myomectomy with scissor morcellation of the leiomyoma within a bag. The specimen was delivered using retrieval bag through the central port under direct vision with the camera inserted through the extended lateral $12 \mathrm{~mm}$ port. Lateral $12 \mathrm{~mm}$ Port was closed with polyglactin on to the rectus sheath, visualizing through the skin incision, using "Langenbeck Retractors". Imme-

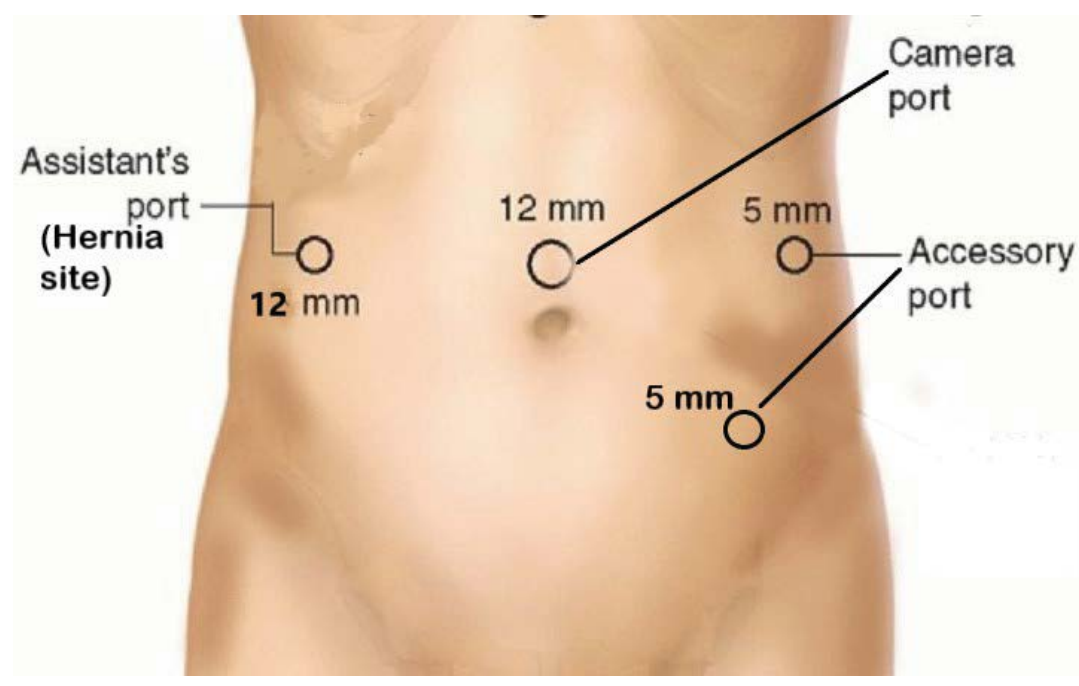

Figure 1: Hernia site and port sites are indicated. Supraumbilical port was chosen to maintain equi-distance between ports, to counteract short abdominopelvic length. Assistant's $5 \mathrm{~mm}$ port extended to place a $12 \mathrm{~mm}$ port to insert the camera. Specimens were delivered through the central port in a retrieval bag. Lateral port rectus sheath was closed with polyglactin.

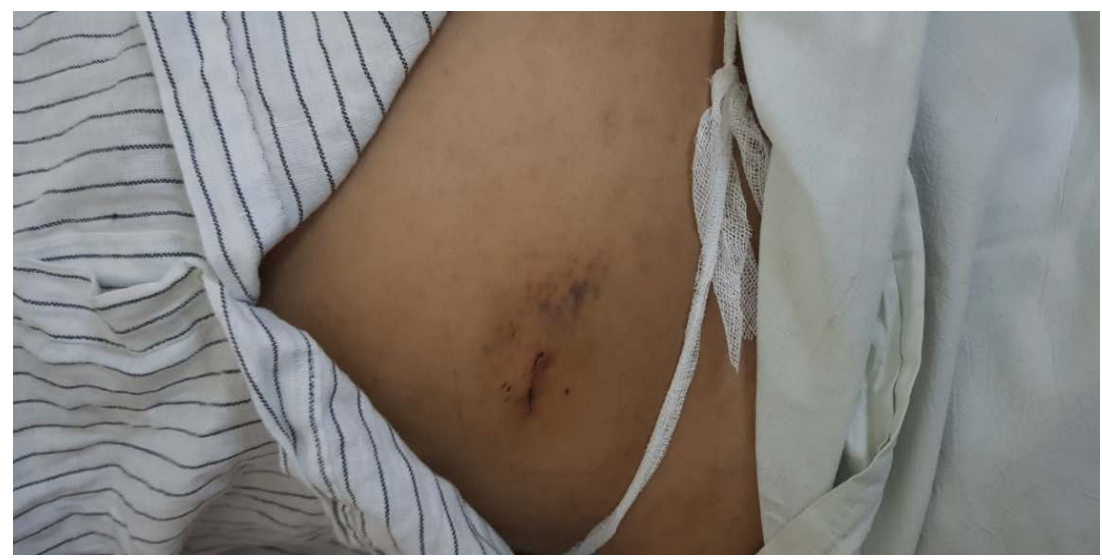

Figure 2: Laparoscopic $12 \mathrm{~mm}$ lateral port site on right lumbar region-no clinically demonstrable hernia at presentation (produced with permission). 
diate Post-op period was uncomplicated \& discharged on day 2 with the plan to be assessed in the clinic for fertility treatment.

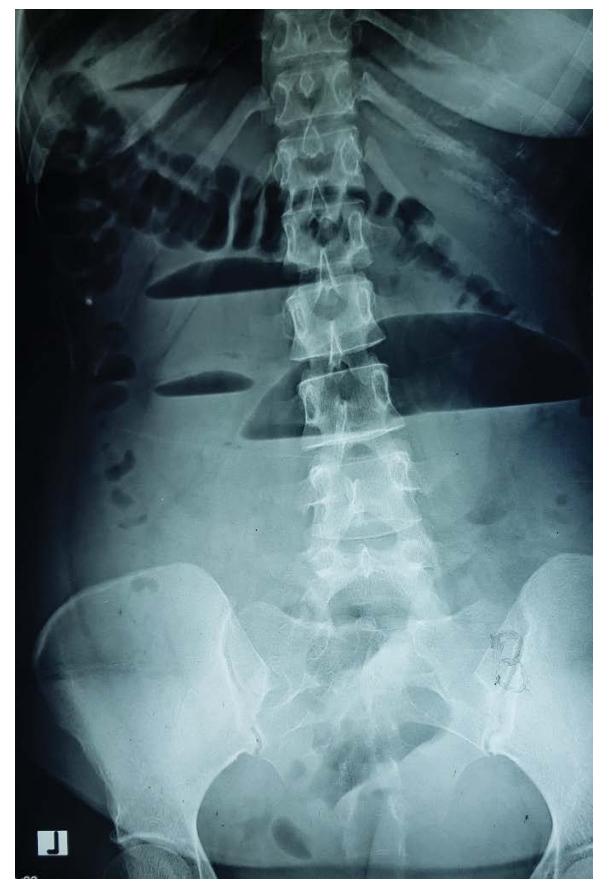

Figure 3: X-Ray supine abdomen. Multiple fluid levels, suggesting small bowel obstruction. No gas under the diaphragm.
She was readmitted on post-operative day three with fever, vomiting, colicky abdominal pain \& bloatedness. Clinical examination revealed peritonism with guarding $\&$ rigidity. Port-site examination was unremarkable with no clinical signs of palpable hernias (Figure 2).

She had deranged white cell count and elevated inflammatory markers (hemoglobin $13.4 \mathrm{~g} / \mathrm{dL}$, white blood cell count of $15.547 / \mathrm{mm}^{3}$, and C-reactive protein of $86 \mathrm{mg} / \mathrm{L}$ ). However, a discrepancy between biochemical markers and the clinical examination findings was noted (not deranged substantially enough as suggestive of suspected bowel injury and peritonitis). She was started on IV antibiotics, as suspected bowel injury leading to peritonitis \& further investigations were carried out. X-ray supine Abdomen showed multiple fluid levels suggestive of small bowel obstruction without gas under the diaphragm (Figure 3). CECT of the abdomen showed a port site hernia with good contrast enhancement, suggesting congested but viable bowel (Figure 4). Immediate laparotomy was resorted into, to release the hernia as well as to assess the integrity and viability of the herniated bowel loop.

Polyglactin stich applied to the sheath at the port closure had untwined, and a clear extended defect of the aponeurosis could be seen in the right ileac fossa with the released stich ligature. Herniation of the small
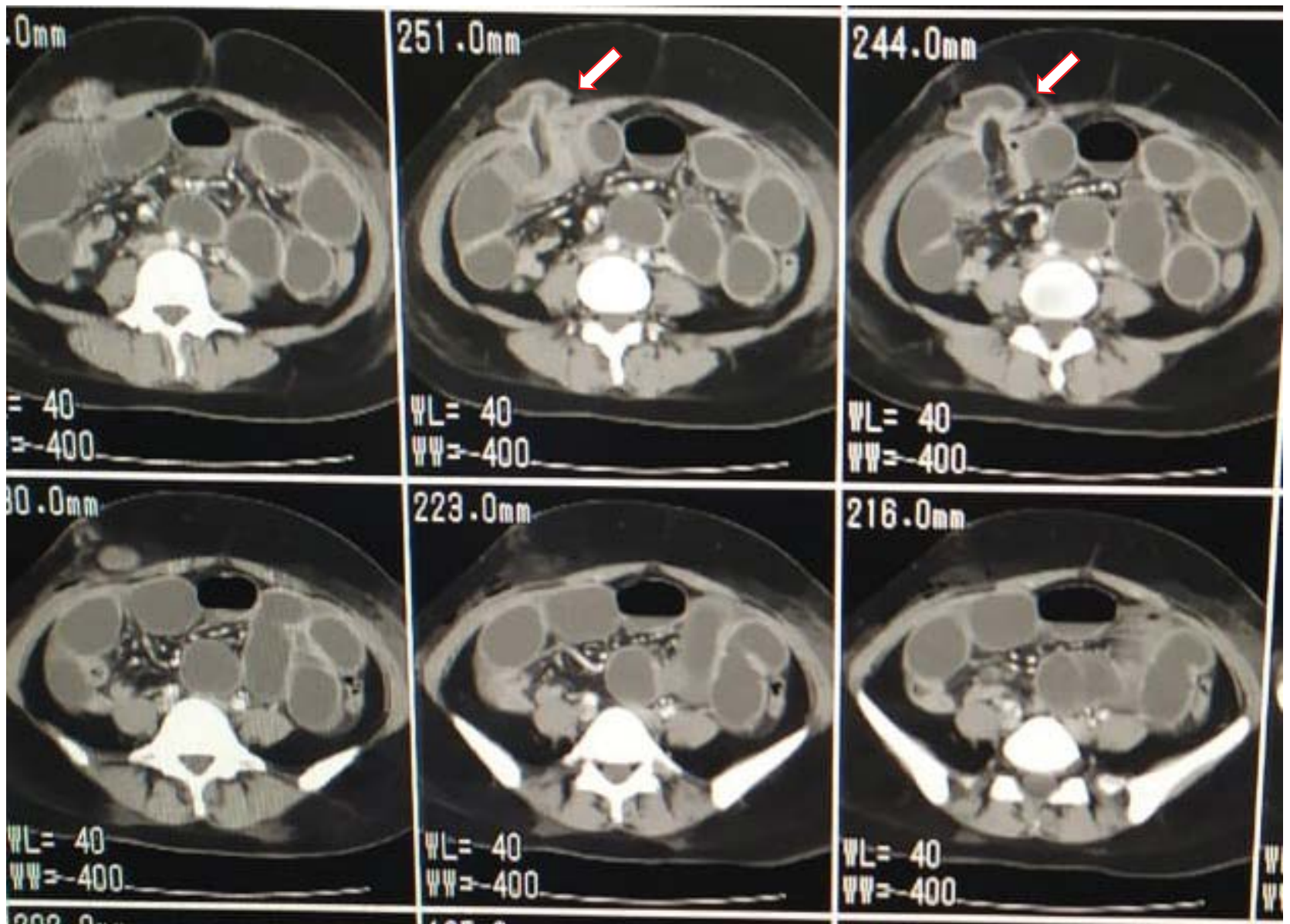

Figure 4: Contrast Enhanced Computed tomography (CECT) scan shows the dilated small bowel protruding into the muscular layer of the abdominal wall (arrow). Good contrast Enhancement suggesting Congested but viable bowel. 
bowel (lleum) in to the anterior abdominal wall, through the rectus sheath defect at the trocar insertion site was noted. After extending the sheath defect, markedly congested bowel was slowly released. Integrity of the bowel was checked with a small bowel survey. Bowel became pinkish and the peristalsis returned after few minutes of observation, confirming viability. Peritoneum was closed with Polyglactin 2-0 and the rectus sheath defect was repaired completely with Proline, from inside. The sub-umbilical midline incision was closed with nylon and 3-0 Monocryl subcuticular sutures applied for the skin. She was observed overnight in the ICU, recovered well with subsiding of fever and rapid improvement of biochemical markers. Discharged from the ward on post op day 3 after debriefing and counselling and is awaiting subfertility treatment.

\section{Discussion}

Trocar site hernia is a complication which can give rise to serious \& life-threatening consequences after a seemingly uncomplicated laparoscopic procedure. TSH is defined as a hernia that occurs through a facial defect created by a laparoscopic port insertion [6]. American Association of Gynecologic Laparoscopists in 1994 has published a large-scale study in a series of $4,385,000$ patients describing the incidence of TSH to be $0.021 \%$ [7]. This is higher than the rate published by some of the newer studies. Boughey, et al. assessed literature and described Richter's hernias at the port site after laparoscopic surgery through a small facial defect rather than the entire bowel wall [2]. It has been shown that larger the wound created by the trocar, greater the risk of TSH. Kadar N, et al. described the incidence of hernia for $10 \mathrm{~mm}$ trocar as $0.23 \%$, rising to $3.1 \%$ for $12 \mathrm{~mm}$ [8]. Depending on the presentation, lino $\mathrm{T}$, et al. classified TSH into 3 types [9].

* Type 1- Early onset \& tends to present within the first 2 weeks with facial plane and peritoneal dehiscence. Small bowel obstruction was commonly observed. In our case scenario she presented on postop day three with intestinal obstruction falling into this category.

* Type 2- Classified as late onset trocar site hernia, when they present after two weeks. They tend to have developed hernial sac with underlying facial defect and intact peritoneum. Only a minor proportion of them would get bowel obstruction.

* Type 3- Progressive wall dehiscence with protruded intestine or omentum following laparoscopic port insertion. They rarely get bowel obstruction and tend to present with whole abdominal wall dehiscence mostly in midline.

According to the severity of bowel obstruction, Symptoms of trocar-site herniation can be varied. TSH diagnosis may be difficult and confusing specially when they present in early post-operative period with varied symptoms. Nausea vomiting and vague abdominal pain can be common after abdominal surgery. If proper vigilance is not exercised, diagnosis can easily be delayed causing bowel to be unviable. In our case, initial clinical picture and biochemical markers were more suggestive of bowel perforation and peritonitis. USS may be less convincing particularly in obese. Diagnosis is commonly aided by plain X-ray film. CECT has significant advantages in differentiating TSH from incarcerated small bowel, ileus \& small bowel obstruction.

Risk factors for TSH have been widely explored by some of the previous studies. They can be widely divided into patient $\&$ surgery related factors. The patient factors include high BMI, Smoking, chronic obstructive pulmonary disease, advanced age over 60 years, uncontrolled diabetes, malnutrition, immune deficiency, port site wound infection, previous abdominal surgeries $[10,11]$. Obesity may be contributing in many ways. Not only it's difficult to close the facial defect properly in obese patients, but also thickened pre-peritoneal space, transversalis facia, omentum and the increased pressure within the abdomen may give rise to facial dehiscence even when proper closure is carried out $[10,11]$. Interestingly, Agaba, et al. suggested that there may be a correlation with sudden weight gain and hernia development after laparoscopy, rather than obesity itself [11]. Presence of a pre-existing umbilical hernia has been identified as a risk factor by many Authors.

Surgery related factors for TSH have been studied and well described. Prolonged surgical time, incision size, excessive force and manipulation of the trocar site (prompting un-noticed enlargement of the facial defect), willful enlargement of the port site to remove a specimen, not closing facial sheath of the lateral ports, size \& type of the trocar used (bladed vs. non bladed), SILS (Single incision laparoscopic surgery) have all been suggested as established risk factors [3,12-14]. However, in our case there were not any identifiable risk factors as above. Diameter and the type of trocar has been reported by few studies. Most of the hernias have occurred when the port is $10 \mathrm{~mm}$ or more. Kadar $\mathrm{N}$, et al. [8] described frequency of hernias to be $0.23 \% \& 3.1 \%$ for $10 \mathrm{~mm} \& 12 \mathrm{~mm}$ trocars respectively extra-umbilical sites. This implies larger the incision, higher the chance of herniation. Use of bladed type trocars are known to cause larger incisions (prompting more hernias) compared to non-bladed types as is shown by Kolata, et al. on pig experimental models [15]. In similarity to indexed case, one precious report described a hernia despite closing the facial defect [16]. Some of the previous reports suggested non bladed trocars may not need port closure [17]. However, we suggest closure of all peripheral ports if they are $7 \mathrm{~mm}$ or more even if non-bladed trocars are used.

Literature review by Shaher Z, assessed different wound closure techniques [18]. Specially designed port closure devices are becoming popular. However old 

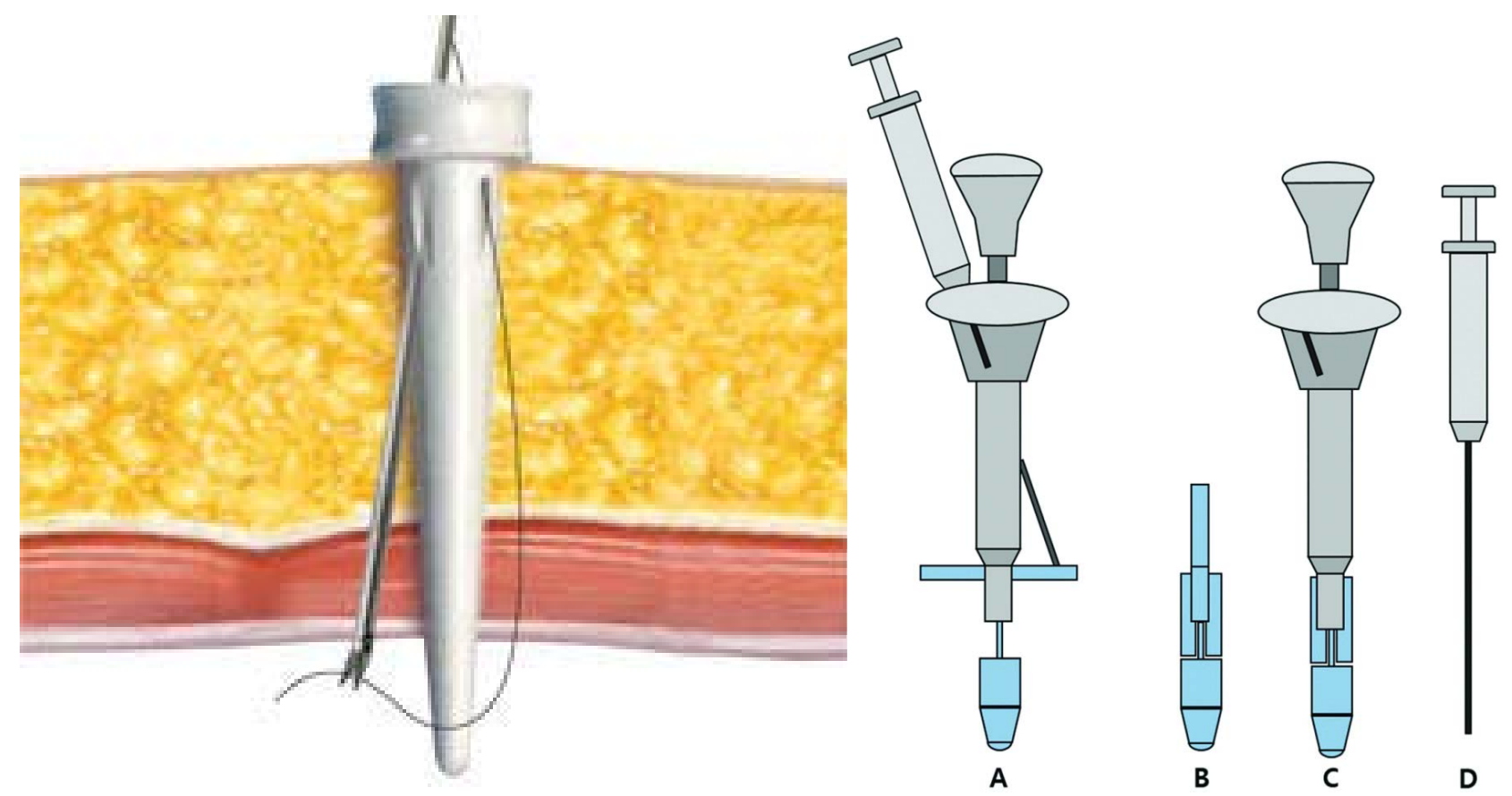

Figure 5: Specially designed port closure devices are faster and may result in fewer complications.

techniques using classical instruments such as "Deschamps needle" and "Veress needle loop technique" can be useful and cost effective as well. We have used "Langenback retractor method" in this case-scenario, which is a natural choice when dedicated devices are not available. Deschamps needle is a rigid noncutting instrument with a handle and a tip that can be passed through the fascial layers. A full thickness closure can be readily accomplished with this instrument. Creating a loop using the Veress needle without any additional instruments is being used in some centers. However, technique may be time consuming to master, even though cost-effective. "Endoclose suture device" uses a spring-loaded suture carrying mechanism and is popular in some settings due to its comparatively law cost and ese of use. Some of the recent studies have assessed the new devices, such as Carter-Thomason needle \& EZ-CloseTM $[19,20]$. This device contains two parts, asuture passer\& a pilot guide. Prospective randomized study evaluating eight different techniques has suggested, the Carter-Thomason device can be faster and resulted in fewer port-closure-related complications [21]. Moreover, radially expanding type trocars are shown to be less painful, have lesser port related complications and may avoid the need for facial closure [22]. However further evaluation may be needed of the above claims. Many conventional as well as newly invented devices and techniques are capable of achieving a satisfactory port closure. However, learning curve, cost-effectiveness, time efficiency \& surgeon's preferability is different in each. A separate evidence review looking in to published studies will be useful to evaluate each of these individual devices in the future (Figure 5).

Use of Polyglactin, PDS, nylon and other suture materials have been studied. Diener, et al. reported a sig- nificantly lower hernia rate for non-absorbable or slowly absorbable sutures in comparison to rapidly absorbable sutures for laparotomy [23]. In our case we have used Polyglactin (Vicryl) which was untwined. However, complication on day 3 makes it unlikely to be related to suture material chosen. We recommend use of none-absorbable or delayed absorbable suture materials with better tensile strength, such as PDS or Prolene compared to polyglactin (early absorbable) for port closure.

\section{Conclusion}

TSH hernia is a devastating complication which may be difficult to diagnose early. It can lead to bowel resection and more sinister complications following a relatively uncomplicated laparoscopic procedure. USS may be less convincing particularly in obese. Diagnosis is commonly aided by plain X-ray film. CECT has significant advantages in differentiating TSH from incarcerated small bowel, ileus \& small bowel obstruction. Royal College of Obstetricians and Gynaecologists guidelines advice closure of all $7 \mathrm{~mm}$ or larger lateral ports and 10 $\mathrm{mm}$ or larger midline ports. It can be prevented with proper pre-op evaluation, employing proper laparoscopic surgical principles, appropriate port selection, good specimen retrieval techniques, and use of proper closure methods \& devices. Conventional J needle, laparoscopic suturing of the rectus sheath under direct vision, port closure devices, all have their own pros \& cons. Specially designed port closure devices are encouraged wherever possible, especially in obese as well as in patience with other risk factors. European hernia society guidelines recommend slowly absorbable monofilament sutures. Suture materials with higher tensile strength such as PDS in comparison to polyglactin may further improve outcome [24]. 


\section{Conflicts of Interest}

The authors declare no conflicts of interests.

\section{References}

1. Reich H, DeCaprio J, Mc Glynn F (1989) Laparoscopic hysterectomy. J Gynecol Surg 5: 213-216.

2. Boughey JC, Notingham JM, Walls AC (2003) Richter's hernia in the laparoscopic era: Four case reports and review of the literature. Surg Laparosc Endosc Percutan Tech 13: 55-58.

3. Fear RE (1968) Laparoscopy: A valuable aid in gynecologic diagnosis. Obstet Gynecol 31: 297-309.

4. Swank HA, Mulder IM, la Chapelle CF, Reitsma JB, Lange JF, et al. (2012) Systematic review of trocar-site hernia. $\mathrm{Br}$ J Surg 99: 315-323.

5. Zhu YP, Liang S, Zhu L, Sun ZJ, Lang JH (2019) Trocarsite hernia after gynecological laparoscopic surgery: A 20-year, single center experience. Chin Med J 132: 2677-2683.

6. Rebibo L, Dhahri A, Chivot C, Cyril C, Yzet T, et al. (2015) Trocar site hernia after laparoscopic sleeve gastrectomy using a specific open laparoscopy technique. Surg Obes Relat Dis 11: 791-796.

7. Montz FJ, Holschneider CH, Munro MG (1994) Incisional hernia following laparoscopy: A survey of the american association of gynecologic laparoscopists. Obstet Gynecol 84: 881-884.

8. Kadar N, Reisch H, Liu CY, Manko GF, Gimpelson R (1993) Incisional hernias after major laparoscopic gynecologic procedures. Am J Obstet Gynecol 168: 1493-1495.

9. lino T, Oishi H, Yamane T, Hirai E, Kameoka S, et al. (2012) A case of incarcerated hernia at the $5 \mathrm{~mm}$ port site after removal of tube drain. SAGES.

10. Bunting DM (2010) Port-site hernia following laparoscopic cholecystectomy. JSLS 14: 490-497.

11. Agaba EA, Rainville H, Ikedila O, Vemulapali P (2014) Incidence of Port-site incisional hernia after single incision laparoscopic surgery. JSLS 18: 204-210.

12. Tonouchi H, Ohmori Y, Kobayashi M, Kusunoki M (2004) Trocar site hernia. Arch Surg 139: 1248-1256.
13. Pereira N, Hutchinson AP, Irani M, Chung ER, Lekovich JP, et al. (2016) 5-millimeter trocar-site hernias after laparoscopy requiring surgical repair. J Minim Invasive Gynecol 23: $505-511$.

14. Margaux JK, Mazdak M, Konstantin Z, Linus C, Monica P (2013) Case report five-millimeter balloon trocar site herniation: Report of two cases and review of literature. J Minim Invasive Gynecol 20: 723-726.

15. Kolata RJ, Ransick M, Briggs L, Baum D (1999) Comparison of wounds created by non-bladed trocars and pyramidal tip trocars in the pig. J Laparoendosc Adv Surg Tech A 9: $455-461$.

16. Margossian H, Pollard RR, Walters MD (1999) Small bowel obstruction in a peritoneal defect after laparoscopic Burch procedure. J Assoc Gynecol Laparosc 6: 343-345.

17. Liu CD, McFadden DW (2000) Laparoscopic port sites do not require fascial closure when non bladed trocars are used. Am Surg 66: 853-854.

18. Shaher Z (2007) Port closure techniques. Surg Endosc 21: 1264-1274.

19. Shetty A, Adiyat KT (2014) Comparison between hand suture and Carter-Thomason needle closure of port sites in laparoscopy. Urol J 11: 1768-1771.

20. Jeon Y, Song S, Han KW, Lee DH, Baek JH (2020) Evaluation of a novel trocar-site closure device in laparoscopic surgery. JSLS 24.

21. Elashry OM, Nakada SY, Wolf JS Jr, Figenshau RS, McDougall EM, et al. (1996) Comparative clinical study of port-closure techniques following laparoscopic surgery. J Am Coll Surg 183: 335-344.

22. Johnson WH, Fecher AM, McMahon RL, Grant JP, Pryor AD (2006) Versa Step trocar hernia rate in unclosed fascial defects in bariatric patients. Surg Endosc 20:1584-1586.

23. Diener MK, Voss S, Jensen K, Buchler MW, Seiler CM (2010) Elective midline laparotomy closure: The inline systematic review and meta-analysis. Ann Surg 251: 843-856.

24. Muysoms FE, Antoniou SA, Bury K, G Campanelli, J Conze, et al. (2015) European hernia society guidelines on the closure of abdominal wall incisions. Hernia 19: 1-24. 